\title{
The Mediterranean diet as a nutrition education, health promotion and disease prevention tool
}

\author{
Suzanne Piscopo* \\ Nutrition, Family and Consumer Studies Office, Faculty of Education, University of Malta, Msida, \\ MSD2080, Malta
}

Submitted January 2009: Accepted May 2009

\begin{abstract}
Objective: While epidemiological research on the protective role of the Mediterranean diet (MD) is highly publicised, little is known about MD education interventions. This exploratory study aimed to provide insight into methodology and effectiveness of the MD as a nutrition education and health promotion tool. Design and setting: Two searches were conducted within PubMed using the terms 'Mediterranean diet' and 'Education' or 'Intervention'. Abstracts yielded were screened for distinct studies in which participants were instructed on the application of MD principles in their diet. Four studies met this criterion. These were further analysed for sample/target population, and intervention design, duration, tools, evaluative measures/tests and outcomes.

Results: Interventions targeted both healthy and at-risk populations and lasted between 12 weeks to 1 year, including follow-up. They used individual counselling, tailored computer-based counselling, group education, internet-based education, cookery classes and printed materials. Outcomes were measured using food diaries, FFQ, questionnaires on psychosocial factors and on usage of the educational tools, as well as anthropometrics and biomarkers. Interventions showed statistically significant increases in participants' intake of vegetables, legumes, nuts, fruit, whole grains, seeds, olive oil and dietary PUFA and MUFA, and statistically significant decreases in total cholesterol, ox-LDL-cholesterol, total:HDL-cholesterol ratio, insulin resistance, BMI, body weight and waist circumference.

Conclusions: MD education interventions may be cost-effective strategies for helping to protect against and treat a variety of health problems in different populations. Details of such interventions need to be publicised internationally. This introductory review could help inform the design of future targeted MD nutrition education.
\end{abstract}

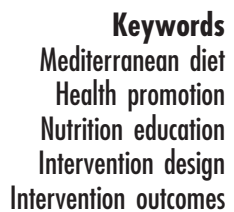

The Mediterranean diet (MD) gained much recognition and worldwide interest in the 1990s as a model for healthful eating habits. This 'popularity' has continued to increase, among both the general public and the scientific community $^{(1-3)}$.

The principles underlying the MD form the basis of different sets of dietary guidelines from around the world, where the emphasis is on increasing intake of vegetables, fruits, nuts, grains, pulses, fish and low-fat dairy products, and opting for monounsaturated fats such as olive oil ${ }^{(4-6)}$. The MD is highly advocated for its health-promoting and disease-preventing characteristics; yet, ironically, during recent decades there has been a gradual abandoning of the MD diet by the populations of the Mediterranean, especially among the younger generations ${ }^{(1,7-14)}$. Simultaneously, while epidemiological research on the protective role of the $\mathrm{MD}$ is highly publicised, little is known about the use and effectiveness of MD education interventions. The purpose of the present paper was to carry out a preliminary exploratory study of published articles on the MD as a nutrition education tool. This was not a full-scale systematic review, but more of an introduction to the phenomenon.

\section{Methodology}

Two online searches were conducted within PubMed citation database using the terms 'Mediterranean diet' and 'Education' or 'Intervention'. The 258 abstracts yielded were screened for distinct studies with a clear 'education' component, education being defined as some form of instruction to participants on the application of MD principles in their diet. Four studies met this criterion and these were further analysed for sample/target population, and educational intervention design, duration, tools, evaluative measures/tests and outcomes. Full details of 


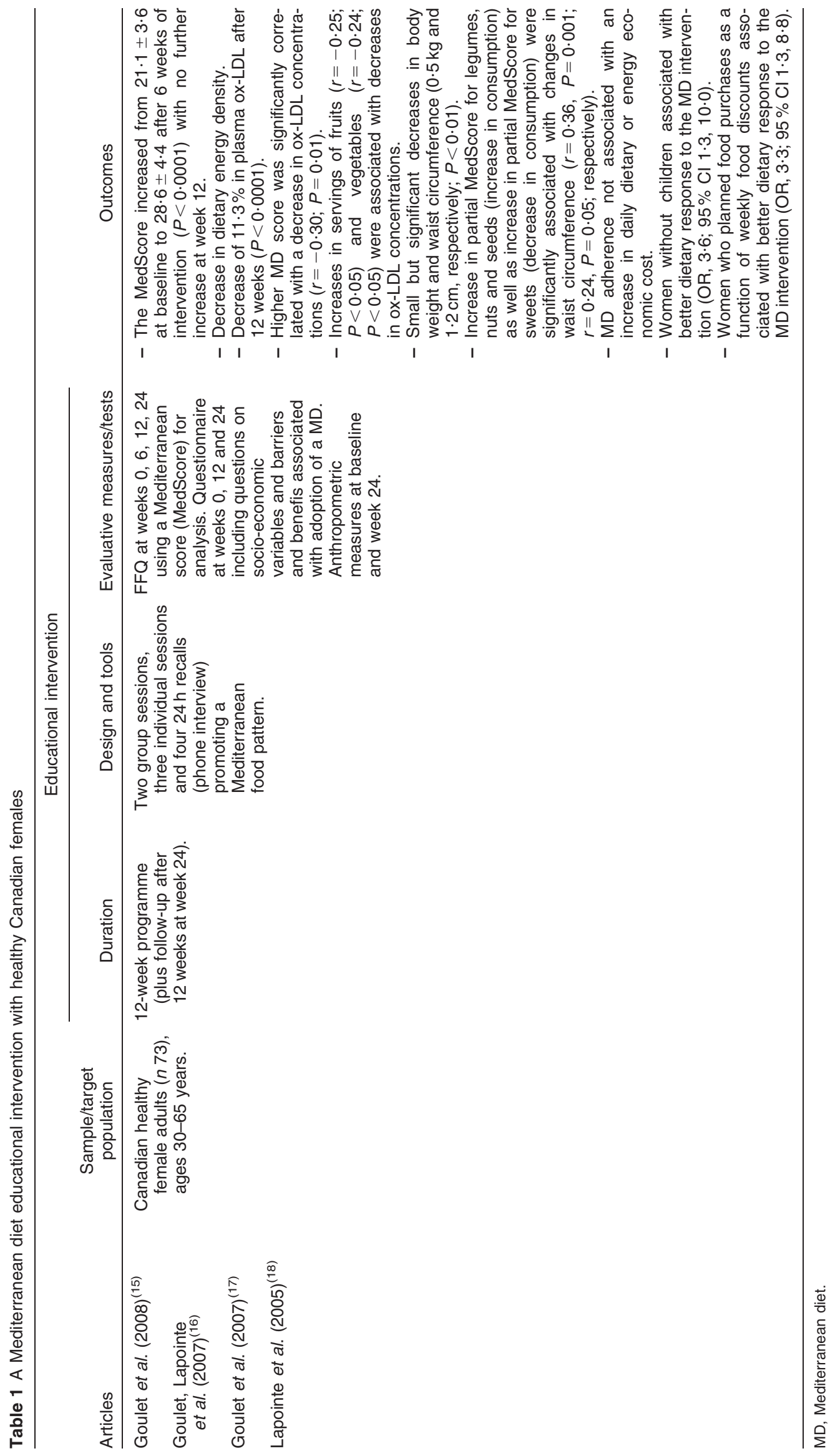




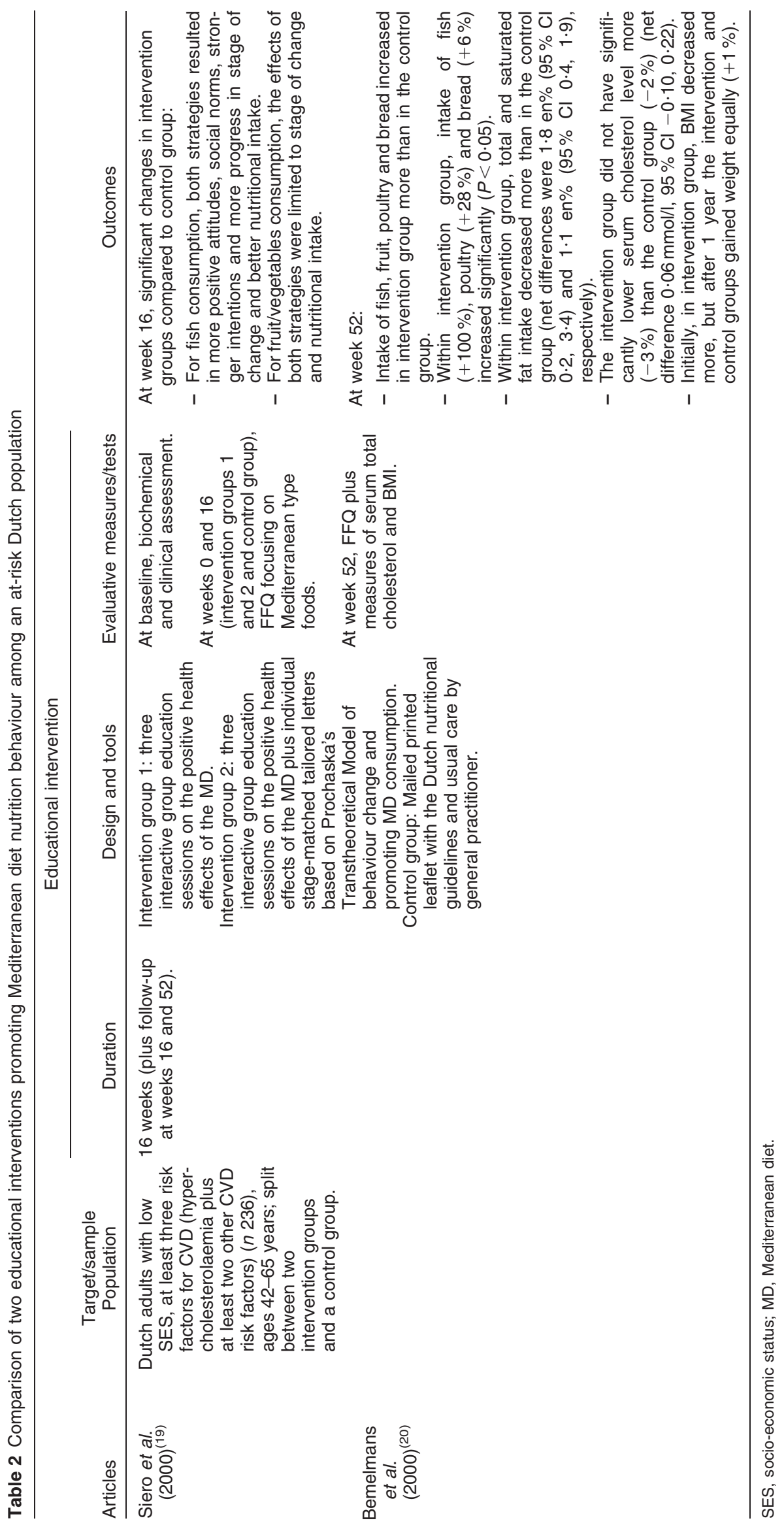


this analysis can be seen in Tables 1-4. A brief summary of each study is given below.

\section{Results}

\section{Study 1}

A group of Canadian healthy female adults participated in a 12-week programme promoting the Mediterranean food pattern $^{(15-18)}$. The programme comprised two group sessions and seven individual sessions with a dietitian (three counselling sessions plus four $24 \mathrm{~h}$ recalls). During the first group session, a dietitian explained the major principles and health benefits of following a MD. Four weeks after the beginning of the intervention, subjects participated in a Mediterranean cooking lesson during which they had to produce a complete meal. Individual sessions took place during weeks 1,6 and 12 to evaluate dietary changes and to select further objectives to increase adherence to the MD. During individual sessions, the dietitian used the FFQ and the Mediterranean food pyramid to promote dietary changes compatible with the participant's food preferences. Unannounced qualitative $24 \mathrm{~h}$ recalls were performed by a dietitian over the telephone at weeks 2, 4, 8 and 10. The objective was to reinforce the key principles of the MD and provide participants with additional support.

Evaluation using FFQ took place at intervals during the programme and 12 weeks after the end of the programme (week 24). Adherence to the programme, also as evidenced by a MD score, led to a small but significant decrease in weight and waist circumference, a reduction in dietary energy density, and was associated with a decrease in circulating oxidised LDL. Adopting a MD pattern of food intake was not associated with increased daily dietary or energy economic cost. Women without children or who used available food discounts to plan their food shopping showed a better dietary response to the MD advice given.

\section{Study 2}

A study with Dutch adults from a socio-economically deprived area, and who had hypercholesterolaemia and at least another two risk factors for CVD, compared the use of two types of MD education interventions (intervention group 1 - group education; intervention group 2 - group education plus individual tailored letter) with a 'care as usual' (control) group ${ }^{(19,20)}$. Both intervention groups participated in three $2 \mathrm{~h}$ interactive group sessions, in which they were briefed on CVD risk factors and on the basic components of the healthy MD; a positive attitude to the MD was encouraged and they were given practical tips on how to adopt a MD food pattern. Booklets with core information from the course were also distributed (or mailed to non-attendees) at the end of each session. A container of diet margarine was also distributed. Members of intervention group 2 also received a Prochaska-based individualised letter in-between group 


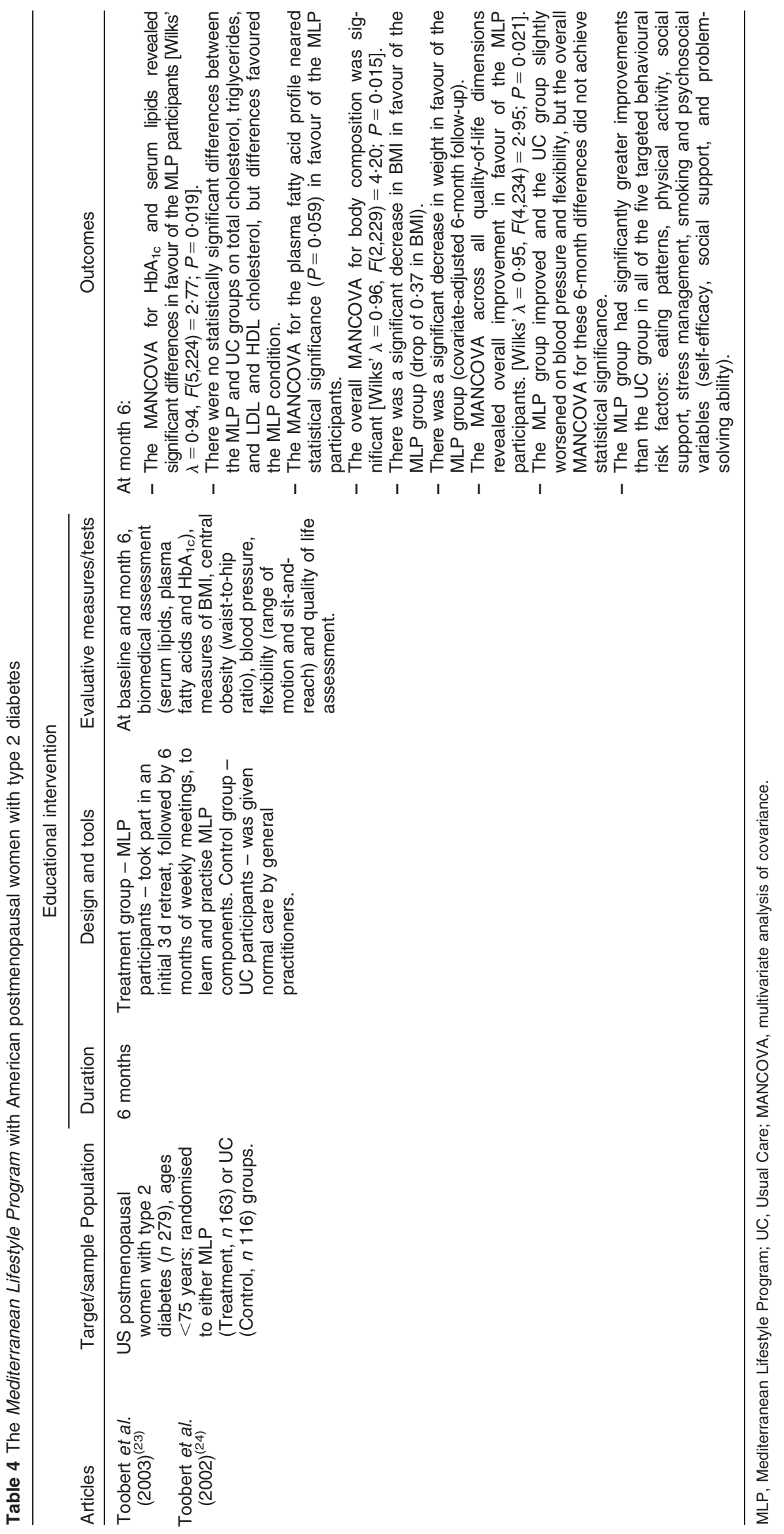


session two and three. The letter contained additional MDrelated information and was tailored to their attitude, selfefficacy, social norm and stage of change. The control group just received a leaflet with the Dutch Nutritional Guidelines and usual care by a general practitioner. The educational aspect of the intervention lasted for 16 weeks.

FFQ that emphasised Mediterranean type foods were administered at baseline and weeks 16 and 52. Biochemical and clinical assessments were conducted at weeks 0 and 52, measuring, amongst others, serum cholesterol, HDL-cholesterol and triglycerides, as well as weight and height. The various results obtained showed that substantial dietary behaviour change can be achieved even by a brief interactive group education intervention on the positive health effects of the MD. Such group education competes in efficacy with individual tailored letters. In fact, there were a number of beneficial changes in dietary habits in the intervention groups compared with the control group, including increased fish, fruit, vegetable and (in the long-term) bread and poultry consumption, and decreases in total fat and saturated fat intake. However, at week 52 the intervention group had not lowered their serum cholesterol levels significantly more than the control group. Moreover, BMI had increased for both intervention and control groups, and total fat and saturated fat intake were still too high.

\section{Study 3}

A study with Scottish healthy females aimed to evaluate the effectiveness of a 6-month internet-based, stepwise, tailored-feedback intervention promoting key components of the $\mathrm{MD}^{(21,22)}$. A Mediterranean Eating website was developed based on various theories including the Health Belief Model, Social Cognitive Theory and the Theory of Planned Behaviour. Aesthetically the website was given a 'Mediterranean feel' and provided healthy eating, shopping and cooking tips, adjusted to reflect and promote the MD. A new set of approximately twenty Mediterranean recipes was added every fortnight. The recipes were inspired primarily by Greek and other Mediterranean dishes. However, recipes for popular Scottish dishes prepared in a Mediterranean style (e.g. with a greater variety and amount of vegetables) were also included. An intervention and a control group were set up. Participants in the intervention group were given information on secure access to the website, and they were emailed weekly and encouraged to visit the website and the updated sections. Eventually, they also received dietary and psychosocial feedback on their food intake. Control group participants received very little feedback and only healthy-eating brochures.

A process evaluation of the website which took place as the intervention progressed indicated that, overall, the majority of the intervention participants perceived the website to be extremely interesting, informative, useful, trustworthy, easy to understand, novel, attractive, encouraging and user-friendly. The recipes section was the most visited. Visits to the website decreased over the duration of the intervention, with lack of time being reported by participants as a prime barrier to using the website on a weekly basis.

Comparative analysis of $7 \mathrm{~d}$ estimated food intake diaries at baseline and at 6 months revealed that the intervention group had significantly increased their intake of vegetables, fruits and legumes, as well as the MUFA:SFA (monounsaturated/saturated fatty acid) ratio in their diet. They also had significantly increased plasma HDL-cholesterol levels and reduced the ratio of total:HDL-cholesterol. The control group also increased their intake of legumes, but saw no other significant beneficial changes compared to baseline. The authors concluded that such an online intervention holds promise in encouraging a greater consumption of plant foods, together with increasing monounsaturated fat and decreasing saturated fat in this type of population.

\section{Study 4}

This study tested the effectiveness of a comprehensive self-management Mediterranean Lifestyle Program (MLP) in reducing cardiovascular risk factors in American postmenopausal women with type 2 diabetes ${ }^{(23,24)}$. Subjects were randomised to a treatment (MLP) or control (usual care (UC)) group. All intervention materials and venues were given a 'Mediterranean feel' and the programme comprised education and training in preparing a Mediterranean-type low-saturated fat diet, stress management, physical activity, smoking cessation (where necessary) and group support skills. The intervention lasted for 6 months: it started with a $3 \mathrm{~d}$ non-residential retreat, followed by weekly $4 \mathrm{~h}$ meetings which consisted of $1 \mathrm{~h}$ each of physical activity, stress management, group Mediterranean potluck dinner and support groups. In the programme, a dietitian individualised carbohydrate and fat intake for each participant in order to optimise blood glucose and lipid concentrations, while still being faithful to the dietary patterns of the MLP.

At month 6 - the end of the intervention - compared to baseline, the MLP treatment group had significantly greater improvements compared with the UC control group on serum lipids, $\mathrm{HbA}_{1 \mathrm{c}}$, BMI, weight and quality of life. They also fared significantly better with respect to physical activity, stress management/coping, smoking cessation and psychosocial variables (self-efficacy, social support and problem-solving ability). The authors proposed that, through the MLP, such a target group can make comprehensive lifestyle changes that may lead to clinically significant improvements in glycaemic control, some CHD risk factors and quality of life.

\section{Discussion}

This brief introductory overview of interventions which had a clear MD education component indicates that such education can have a positive impact on food intake and, 
consequently, strong potential for reducing health risk factors. The interventions targeted both healthy and atrisk populations and lasted between 12 weeks to 1 year, including follow-up. The MD education component used individual counselling, tailored computer-based counselling, group education, internet-based education, cookery demonstrations and classes, and take-home printed materials. Outcomes studied included adherence to the MD diet and its impact or relation to body weight, waist-to-hip ratio and lipid profiles. Outcomes were measured using food diaries and FFQ, often using MD scores to assess adherence to the diet, questionnaires on psychosocial factors and on usage of the educational tools, as well as anthropometrics and biomarkers. Interventions showed statistically significant increases in intake of vegetables, legumes, nuts, fruit, whole grains, seeds, olive oil and dietary PUFA and MUFA, and statistically significant decreases in total cholesterol, ox-LDL-cholesterol, total:HDL-cholesterol ratio, insulin resistance, BMI, body weight and waist circumference.

In two of the studies, it was also proposed that using MD nutrition education may be a relatively cost-effective strategy for improving health and reducing health-risk factors. Similarly, in a recent study in Australia, which evaluated the economic performance of ten nutrition interventions, MD intervention emerged among the most cost-effective, where economic performance was expressed as cost per QALY (quality adjusted life year) gained ${ }^{(25)}$. The authors concluded that such nutrition interventions can constitute a highly efficient component of a strategy to reduce the growing disease burden linked to over- or under-nutrition.

This brief overview also reinforces the proposition made earlier that there seems to be a gap in the literature with respect to implementation and evaluation of MD education interventions. The four studies reported all took place outside the Mediterranean region. Little seems to be known internationally on how Mediterranean nations themselves are promoting the MD in their public health campaigns. It is likely that many interventions are carried out, but are not reported beyond the countries' borders. For example, in the early 2000s, the then Maltese Health Promotion Department published a leaflet on the value of the MD which it often used as a basis for presentations in different community settings and which was disseminated widely through health fairs, in schools and from its outreach offices. The Department also launched a Cancer Prevention recipe book that included recipes following the MD approach ${ }^{(26)}$. In addition, traditional Maltese healthy foods are regularly promoted during Home Economics lessons in Maltese schools. Interestingly, a study with Cretan primary school children concluded that attempts to introduce the principles of the MD to children through nutrition education requires innovative, enthusiastic and highly motivated teachers ${ }^{(27)}$.

Undoubtedly, one can adopt various channels and media for using the MD as a nutrition education and health promotion tool. The four studies reported earlier used a variety of media and, even when there was little face-to-face interaction, personalisation made a difference. Essentially, messages are more effective if they are based on theory, targeted, made easily accessible and their applicability is clear for the intended audience. Even food labelling may be a vehicle for promoting the MD. Recently, the US-based Oldways group launched the Mediterranean Mark for food packaging as a 'gold standard for healthy eating' and to help consumers identify 'Med Diet food' ${ }^{(28)}$. Such labelling could serve as a valuable point-of-purchase educational tool complementary to other MD education strategies.

\section{Conclusion}

MD education interventions may help protect against and treat a variety of health problems in different populations. More extensive research is required to review interventions which have used MD education and strategies which were effective in bringing about positive health behaviour change. Mediterranean countries themselves need to publicise the outcomes of their campaigns in international fora and scientific publications. In the meantime, the results of this introductory review could help inform the choice and design of future MD nutrition education for specific target populations.

\section{References}

1. Alexandratos N (2006) The Mediterranean diet in a world context. Public Health Nutr 9, 111-117.

2. Regmi A, Ballenger N \& Putnam J (2004) Globalisation and income growth promote the Mediterranean diet. Public Health Nutr 7, 977-983.

3. Lairon D (2007) Intervention studies on Mediterranean diet and cardiovascular risk. Mol Nutr Food Res 51, 1209-1214.

4. WHO Regional Office for Europe (2000) CINDI Dietary Guide. Copenhagen: WHO Regional Office for Europe.

5. Food Standards Agency (UK) (2007) The eatwell plate. http:// www.eatwell.gov.uk/healthydiet/eatwellplate/ (accessed 15 February 2008)

6. United States Department of Agriculture (2005) My pyramid. http://www.mypyramid.gov/index.html (accessed 15 February 2008).

7. Belahsen R \& Rguibi M (2006) Population health and Mediterranean diet in southern Mediterranean countries. Public Health Nutr 9, 1130-1135.

8. Piscopo S (2005) Global versus local: a socio-ecological study of the westernisation of Maltese children's diets. Paper presented at the International Conference of the Institute of Consumer Sciences. England: Liverpool John Moores University.

9. Tur JA, Romaguera D \& Pons A (2004) Food consumption patterns in a Mediterranean region: does the Mediterranean diet still exist? Ann Nutr Metab 48, 193-201.

10. Trichopoulou A, Costacou T, Barnia C \& Trichopoulos D (2003) Adherence to a Mediterranean diet and survival in a Greek population. New Eng Jour Med 348, 2599-2608.

11. Moreno LA, Sarria A \& Popkin BM (2002) The nutrition transition in Spain: a European Mediterranean country. Eur Journal of Clin Nutr 56, 992-1003. 
12. Tessier S \& Gerber M (2002) Understanding the loss of the maintenance of the Mediterranean diet: a comparative study of the determinants of food habits and preferences between Sardinia and Malta. Paper presented at the Xth Food Choice Conference. The Netherlands: University of Wageningen.

13. Kafatos A, Verhagen H, Moschandreas J, Apostolaki I \& van Westerop JJM (2000) Mediterranean diet of Crete: foods and nutrient content. J Am Diet Assoc 100, 1487-1493.

14. Greco L, Musmarra F, Franzese C \& Auricchio S (1998) Early childhood feeding practices in southern Italy: is the Mediterranean diet becoming obsolete? Study of 450 children aged 6-32 months in Campania, Italy. Acta Paediatr 87, 250-256.

15. Goulet J, Lamarche B \& Lemieux S (2008) A nutritional intervention promoting a Mediterranean food pattern does not affect total daily dietary cost in North American women in free-living conditions. J Nutr 138, 54-59.

16. Goulet J, Lapointe A, Lamarche B \& Lemieux S (2007) Effect of a nutritional intervention promoting the Mediterranean food pattern on anthropometric profile in healthy women from the Québec city metropolitan area. Eur J Clin Nutr 61, 1293-1300.

17. Goulet J, Lamarche B \& Lemieux S (2007) Factors influencing the dietary response to a nutritional intervention promoting the Mediterranean food pattern in healthy women from the Québec City metropolitan area. Health Educ Res 22, 718-726.

18. Lapointe A, Goulet J, Couillard C, Lamarche B \& Lemieux S (2005) A nutritional intervention promoting the Mediterranean food pattern is associated with a decrease in circulating oxidized LDL particles in healthy women from the Québec City metropolitan area. J Nutr 135, 410-415.

19. Siero FW, Broer J, Bemelmans WJ \& Meyboom-de Jong BM (2000) Impact of group nutrition education and surplus value of Prochaska-based stage-matched information on health-related cognitions and on Mediterranean nutrition behavior. Health Educ Res 15, 635-647.

20. Bemelmans WJ, Broer J, de Vries JH, Hulshof KF, May JF \& Meyboom-De Jong B (2000) Impact of Mediterranean diet education versus posted leaflet on dietary habits and serum cholesterol in a high risk population for cardiovascular disease. Public Health Nutr 3, 273-283.

21. Papadaki A \& Scott JA (2006) Process evaluation of an innovative healthy eating website promoting the Mediterranean diet. Health Educ Res 21, 206-218.

22. Papadaki A \& Scott JA (2005) The Mediterranean eating in Scotland experience project: evaluation of an internetbased intervention promoting the Mediterranean diet. Br J Nutr 94, 290-298.

23. Toobert DJ, Glasgow RE, Strycker LA, Barrera M Jr, Radcliffe JL, Wander RC \& Bagdade JD (2003) Biologic and quality-of-life outcomes from the Mediterranean Lifestyle Program: a randomized clinical trial. Diabetes Care 26, 2288-2293.

24. Toobert DJ, Strycker LA, Glasgow RE, Barrera M \& Bagdade JD (2002) Enhancing support for health behavior change among women at risk for heart disease: the Mediterranean Lifestyle Trial. Health Educ Res 17, 574-585.

25. Dalziel K \& Segal L (2007) Time to give nutrition interventions a higher profile: cost-effectiveness of 10 nutrition interventions. Health Promot Int 22, 271-283.

26. Department of Health Promotion (Malta) (2003) Cancer prevention recipe book. http://www.sahha.gov.mt/pages. aspx?page $=324$ (accessed 15 February 2008).

27. Kafatos I, Peponaras A, Linardakis M \& Kafatos A (2004) Nutrition education and Mediterranean diet: exploring the teaching process of a school-based nutrition and media education project in Cretan primary schools. Public Health Nutr 7, 969-975.

28. Mediterranean Foods Alliance and Oldways (2007) The Mediterranean mark. http://mediterraneanmark.org/The MedMark2.htm (accessed 15 February 2008). 